\begin{tabular}{|c|}
\hline $\begin{array}{r}\text { PRAMANA } \\
- \text { journal of } \\
\text { physics }\end{array}$ \\
\hline
\end{tabular}

\title{
Leptonic minimal flavour violation in warped extra dimensions
}

\author{
ABHISHEK M IYER* and SUDHIR K VEMPATI \\ Centre for High Energy Physics, Indian Institute of Science, Bangalore 560 012, India \\ *Corresponding author. E-mail: abhishek@cts.iisc.ernet.in
}

\begin{abstract}
Lepton mass hierarchies and lepton flavour violation are revisited in the framework of Randall-Sundrum models. Models with Dirac-type as well as Majorana-type neutrinos are considered. The five-dimensional $c$-parameters are fit to the charged lepton and neutrino masses and mixings using $\chi^{2}$ minimization. Leptonic flavour violation is shown to be large in these cases. Schemes of minimal flavour violation are considered for the cases of an effective LLHH operator and Dirac neutrinos and are shown to significantly reduce the limits from lepton flavour violation.
\end{abstract}

Keywords. Randall-Sundrum; minimal flavour violation.

PACS Nos 11.30.Rd; 13.40.Gp

\section{Introduction}

The large hierarchy between the Planck scale $\left(\sim 10^{18} \mathrm{GeV}\right)$ and the electroweak scale $\left(\sim 10^{2} \mathrm{GeV}\right)$ is one of the biggest mysteries of particle physics. RS model proposes a solution to this problem in terms of an extra space dimension compactified on $S_{1} / Z_{2}$, with a large negative bulk cosmological constant [1]. In this scenario there are two branes, referred to as IR or the SM brane and UV or the Planck brane situated at different points of the extra dimension. The geometry of the bulk is warped such that the corrections to the Higgs mass, sensitive to the Planck scale, are exponentially suppressed. By allowing the fermions to propagate in the bulk, one can also explain the Standard Model mass hierarchy problem. In the present work, we revisit the question of lepton mass hierarchies in the RS model by fitting the five-dimensional bulk masses to the leptonic data. We consider models with both Dirac and Majorana-type neutrinos. We then discuss schemes of minimal flavour violation as a solution to the flavour problem. Finally, let us note that the metric for the RS model is $\mathrm{d} s^{2}=\mathrm{e}^{-2 \sigma(y)} \eta_{\mu \nu} \mathrm{d} x^{\mu} \mathrm{d} x^{\nu}-\mathrm{d} y^{2}$, where $\sigma(y)=k y$ and $R$ is the compactification radius. We work in the scenario where the Higgs is localized on the IR boundary. 


\section{Neutrino mass models in RS}

(a) The LLHH case: The Majorana case is considered as an effective LHLH operator. In this case, the $5 \mathrm{D}$ action is given by

$$
\begin{aligned}
S= & \int \mathrm{d}^{4} x \int \mathrm{d} y \sqrt{-g}\left(\frac{\kappa}{\Lambda^{(5)}} L H L H+Y_{\mathrm{E}} \bar{L} E H\right) \delta(y-\pi R) \\
& +\int \mathrm{d}^{4} x \int \mathrm{d} y \sqrt{-g}\left(\bar{L}\left(i \not D-m_{\mathrm{L}}\right) L+\bar{E}\left(i \not D-m_{\mathrm{E}}\right) E\right),
\end{aligned}
$$

where $\Lambda^{(5)}=10^{18}$ is the fundamental five-dimensional reduced Planck scale. The bulk masses are expressed as $m_{a}=c_{a} \sigma^{\prime}$ where $a=L, E . c_{a}$ are $3 \times 3$ matrices in flavour space. The zero mode mass matrix for the leptons is given by

$$
\begin{aligned}
& \left(m_{v}\right)_{i, j}=\tilde{\kappa}_{i, j}^{(0,0)} \frac{v^{2}}{2 \Lambda^{(5)}} \mathrm{e}^{2 k r \pi} f_{i}^{\mathrm{L}}\left(c_{\mathrm{L}}^{i}\right) f_{j}^{\mathrm{L}}\left(c_{\mathrm{L}}^{j}\right), \\
& \left(M_{\mathrm{E}}\right)_{i j}=\frac{v}{\sqrt{2}}\left(\tilde{Y}_{\mathrm{E}}^{(5)}\right)_{i j} f_{i}^{\mathrm{L}}\left(c_{\mathrm{L}}^{i}\right) f_{j}^{\mathrm{R}}\left(c_{\mathrm{E}}^{j}\right),
\end{aligned}
$$

where $i, j$ are the flavour indices. $\tilde{\kappa}_{i j}^{(0.0)}=2 k \kappa_{i j}$ and $\left(\tilde{Y}_{\mathrm{E}}^{(5)}\right)_{i j}=2 k\left(Y_{\mathrm{E}}\right)_{i j}$ are the order-one Yukawa couplings. The bulk profiles for the zero mode fermions is given by [2,3] $f_{i}\left(c_{a}^{i}\right)=N\left(c_{a}^{i}\right) \mathrm{e}^{-c_{a}^{i} \sigma}$, where the normalization constant is flavour-dependent and is given by $N\left(c_{a}^{i}\right)=\left(\left(0.5-c_{a}^{i}\right) /\left(\mathrm{e}^{\left(1-2 c_{a}^{i}\right) k \pi r}-1\right)\right)^{1 / 2}$. The form of the profiles is the same for both $\mathrm{L}$ and $\mathrm{R}$.

(b) Dirac case: In this case, the action is given by

$$
S=\int \mathrm{d}^{4} x \int \mathrm{d} y \sqrt{-g}\left(Y_{\mathrm{N}} \bar{L} N \tilde{H} \delta(y-\pi R)+\bar{N}\left(i \not D-m_{N}\right) N\right)+\cdots,
$$

where ... are the remaining terms in eq. (1). The zero mode mass matrix in this case is given as

$$
\left(M_{a}\right)_{i j}=\frac{v}{\sqrt{2}}\left(\tilde{Y}_{a}^{(5)}\right)_{i j} f_{i}^{\mathrm{L}}\left(c_{\mathrm{L}}^{i}\right) f_{j}^{\mathrm{R}}\left(c_{a}^{j}\right),
$$

where $\left(\tilde{Y}_{a}^{(5)}\right)_{i j}=2 k\left(Y_{a}\right)_{i j}$ and $a=E, N$.

Results: The zero mode profiles are fits to leptonic data including both charged leptons and neutrino masses. The details of the fit are given in [4]. In figure 1 we show regions of the parameter space in the $c_{E^{1}}-c_{E^{3}}$ plane for both the LLHH case and the Dirac case. There are similar regions in $c_{E^{1}}-c_{E^{2}}$ space. The order-one Yukawa couplings are chosen appropriately between 0.5 and 4 . For the LLHH case, $c_{\mathrm{L}} \approx 0.9$ for all three generations while for the Dirac case $c_{\mathrm{L}} \sim 0.5-0.6$ from the third to the first generation.

The strongest leptonic flavour violating limit is given by $\mu \rightarrow e \gamma$ [5]. The branching ratio is given by

$$
\mathrm{BR}(\mu \rightarrow e \gamma)=\frac{1}{\left(G_{\mathrm{F}} m_{\mu}^{2}\right)^{2}}\left(\left|C^{\mathrm{L}}\right|^{2}+\left|C^{\mathrm{R}}\right|^{2}\right) .
$$



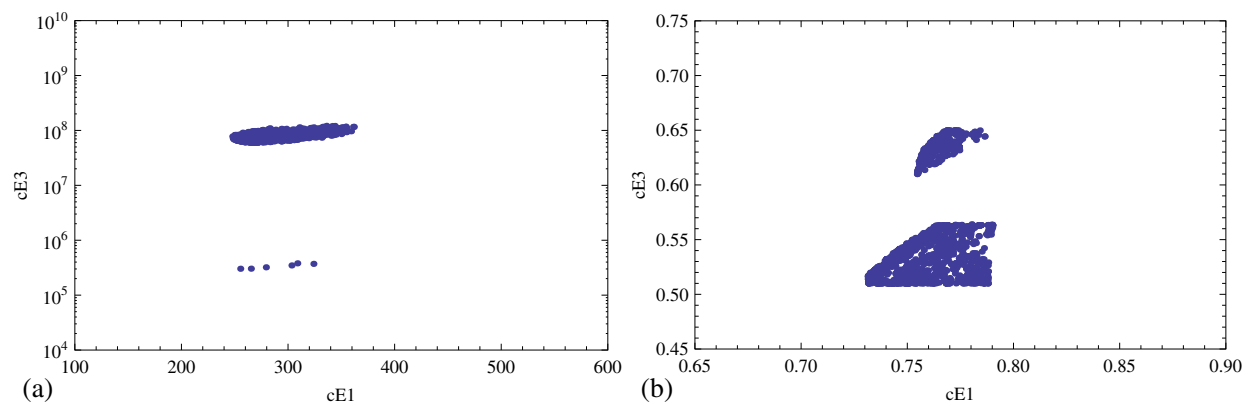

Figure 1. The panel (a) corresponds to the LLHH case. It shows regions of the parameter space in the $c_{E^{1}}-c_{E^{3}}$ plane. One can see the large hierarchy between the first and the third generation charged singlet masses. Panel (b) shows the same plot for Dirac neutrinos. Here, the charged singlets are of the same order for all three generations.

The Wilson coefficients $C^{\mathrm{L}, \mathrm{R}}$ are given as

$$
C^{\mathrm{L}, \mathrm{R}}=\frac{m_{\mu}^{2}}{16 \pi^{2}} \frac{\left(Y_{\mathrm{E}} k\right)^{2}}{M_{\mathrm{KK}}^{2}}\left(U^{\mathrm{L}, \mathrm{R}}\right)_{12},
$$

where $U^{\mathrm{L}, \mathrm{R}}$ is the rotation matrix from the flavour basis to the mass basis and are given as $U_{i, j}^{\mathrm{L}}=f_{i}^{\mathrm{L}}\left(c_{\mathrm{L}}^{i}\right) / f_{j}^{\mathrm{L}}\left(c_{\mathrm{L}}^{j}\right)$ and $U_{i, j}^{\mathrm{R}}=f_{i}^{\mathrm{R}}\left(c_{\mathrm{E}}^{i}\right) / f_{j}^{\mathrm{R}}\left(c_{\mathrm{E}}^{j}\right)$, where $i<j$. We can infer that, since the elements of $U^{\mathrm{L}}$ for both the LLHH and Dirac cases, have order one entries, there is significant misalignment between the flavour and the mass basis. Thus, one requires a Kaluza-Klein scale of $\gtrsim 50 \mathrm{TeV}$ to keep the branching ratio within the experimental limits.

Minimal flavour violation: We invoke minimal flavour violation [6,7] and extend it to RS [8]. With the minimal field content, the Standard Model flavour symmetry group is $S U(3)_{1} \equiv S U(3)_{\mathrm{L}} \times S U(3)_{\mathrm{E}}$ in the leptonic sector. Under this ansatz, the Yukawas are promoted to spurions and have specific transformation under the SM flavour group. The bulk masses are aligned with the Yukawas so as to remove alternate sources of flavour violation. Using this ansatz we can rotate away the contribution due to charge lepton KK mixing. The branching fraction is given by

$$
\mathrm{BR}(\mu \rightarrow e \gamma)=\frac{1}{\left(G_{\mathrm{F}} m_{\mu}^{2}\right)^{2}}\left(\frac{m_{\mu}^{2}}{16 \pi^{2} M_{\mathrm{KK}}^{2}}\right)^{2}\left(\tilde{\kappa}^{(0,0)} \kappa^{(0,0)^{\dagger}}\right)_{12}^{2} .
$$

This brings down the KK mass scale to around $3 \mathrm{TeV}$ while keeping the branching fractions under experimental bounds. For the Dirac case, the flavour group $S U(3)_{1}$ is augmented by $S U(3)_{\mathrm{N}}$. The expression for the branching fraction in this case is similar to eq. (4) with $\tilde{\kappa}^{(0,0)}$ replaced by $\tilde{Y}_{\mathrm{N}}$.

Summary: Minimal flavour violation is a powerful technique to reduce the large leptonic flavour violation present in RS models with bulk fermions. While the present work discussed the effective operator and the Dirac case, similar analysis can be made for the bulk Majorana case. The details are discussed in [4]. 


\section{Abhishek M Iyer and Sudhir K Vempati}

\section{References}

[1] L Randall and R Sundrum, Phys. Rev. Lett. 83, 3370 (1999), arXiv:hep-ph/9905221

[2] T Gherghetta and A Pomarol, Nucl. Phys. B586, 141 (2000), arXiv:hep-ph/0003129

[3] S J Huber and Q Shafi, Phys. Lett. B498, 256 (2001), arXiv:hep-ph/0010195

[4] Abhishek M Iyer and Sudhir K Vempati, Phys. Rev. D (to appear), arXiv:hep-ph/1206.4383

[5] New limit on the lepton-flavour violating decay $\mu \rightarrow e$ gamma, hep-ex: arXiv:1107.5547

[6] G D’Ambrosio, G F Giudice, G Isidori and A Strumia, Nucl. Phys. B645, 155 (2002), hep$\mathrm{ph} / 0207036$

[7] V Cirigliano, B Grinstein, G Isidori and M B Wise, Nucl. Phys. B728, 121 (2005), arXiv:hep-ph/0507001 [hep-ph]

[8] A L Fitzpatrick, L Randall and G Perez, Phys. Rev. Lett. 100, 171604 (2008) 